\title{
ESTABILIDAD DEL RENDIMIENTO EN HÍBRIDOS DE MAÍZ POR DIFERENTE ORDEN DE CRUZA EN LA PRODUCCIÓN DE SEMILLA ${ }^{1}$
}

\author{
Alejandro Espinosa ${ }^{2}$, Margarita Tadeo ${ }^{3}$, Angel Piña del Valle ${ }^{4}$
}

\begin{abstract}
RESUMEN
Estabilidad del rendimiento en híbridos de maíz por diferente orden de cruza en la producción de semilla. Cuando hay asincronía en la floración de los progenitores de un híbrido doble, puede utilizarse la técnica "criss cross", que es el cruce intercambiado de líneas a fin de eliminar el diferencial a floración; como ocurre con el híbrido doble de maíz H-137 para Valles Altos de México (2200 a 2350 msnm). En este maíz, con esta técnica, se facilita el proceso de produción de semilla y disminuye la asincronia a floración. En este trabajo se evaluaron cinco diferentes combinaciones del híbrido H-137 así como dos híbridos trilineales obtenidos con tres de las cuatro líneas del híbrido doble señalado. Con el objetivo de definir la capacidad productiva y estabilidad de los diferentes órdenes de cruza. Se establecieron tres experimentos: Dos en Chapingo, México, y uno en Cuautitlán, México, ubicados a $2240 \mathrm{msnm}$. Se aplicaron los parámetros de estabilidad de Eberhart y Russell (1966), definiéndose los siete genotipos como "estables". el orden de cruza original (M37xM36) x (M 17xM 18), rindió 5,32 tha y fue superado numéricamente por todas las combinaciones alternas. La cruza inversa (M17xM18) x (M37xM36) superó en $13,2 \%$ y la cruza (M36 xM17) x (M37xM 18) en $16,4 \%$ a la cruza original, sin embargo cstadísticamente fueron similares todas las combinaciones. Los dos híbridos trilineales superaron estadísticamente el resto de los materiales. Uno de ellos rindió 6,99 t/ha (MI 7xM18) x M36 y 7,48 tha el otro (M17xM18) x M37.
\end{abstract}

\begin{abstract}
Yield stability of coro (Zea mays L.) by different crossing order of seed production. When there is not a synchronized blooming of the progenitors of a double hybrid, the "criss cross" technique can be used, which consist of interchanging lines in a cross to eliminate the blooming differences; as it happens to the H-137 double corn hybrid for the High Mexican Valleys (2200 to 2350 masl). This technique is used with this corn to ease the seed production process and to lessen the blooming differential. Five different combinations of the $\mathrm{H}-137$ hybrid, as well as two trilineal hybrids (obtained with three out of the four lines of the mentioned double hybrid) were evaluated in this trial. The main objective was to define the productive capacity and stability of the different crossing orders. Three assays were conducted: two in Chapingo and one in Cuautitlan, Mexico; located at 2240 masl. The stability parameters of Eberhart and Russell (1966) were applied, defining the seven genotypes as 'stables'. The original crossing order (M37x M36) x (M17xM18) yielded 5.32 tha and was numerically surpassed by all the other alternating combinations. The inverse crosses (M17xM18) x (M37xM38) and (M36xM 17) $\mathrm{x}(\mathrm{M} 37 \mathrm{xM} 18)$ surpassed the original cross by $13.2 \%$ and $16.4 \%$, respectively; although all the combinations were statistically similar. The two trilineal hybrids statistically surpassed all other crosses.
\end{abstract}

\section{INTRODUCCION}

La coincidencia en la floración de los progenitores es muy importante en maíces híbridos, ya que de ella depende una buena producción de semilla. La asincronía en la floración hace que se dificulte el mantenimiento de la calidad genética; lo mejor es la coincidencia total que minimice la contaminación con polen extraño (Asteinza et $a l, 1990)$.

\footnotetext{
1 Presentado en la XL Reunión Anual del PCCMCA en Costa Rica, América Central. 13 al 19 de marzo, 1994.

2 Investigador Titular, Tecnología de Semillas, CEV AMEX. INIFAP. SARG y Profesor de Ingeniería Agrícola, FESC-UNAM, México.

3 Profesora de Ingeniería Agrícola, FESC-UNAM, México

4 Profesor de Ingeniería Agrícola, FESC-UNAM, México
} 
Sin embargo, frecuentemente se presenta cierto diferencial ya que en el mejoramiento genético realizar cruzamientos para formar híbridos se utilizan materiales heterogéneos entre sí, para aprovechar el alto nivel de heterosis; en muchas ocasiones al realizar los cruzamientos entre líneas, se presenta un diferencial de varios días, lo cual complica la producción de la semilla, teniendo que recurrirse a prácticas agronómicas para reducir ese diferencial y conseguir que la producción máxima de polen en el progenitor masculino coincida con la aparición de los estigmas en el progenitor femenino (Tadeo, 1991). Entre las prácticas agronómicas utilizadas para promover sincronia se puede señalar: Densidad de población, fertilización, aplicación de riegos, podas, aplicación de fitohormonas, etc. No obstante, la técnica, más utilizada para disminuir la asincronía es la siembra diferencial entre los progenitores; pero este tipo de siembra implica incremento en los costos de producción, ya que se duplican labores y se dificulta el manejo agronómico.

Una posibilidad económica para lograr coincidencia total, es el empleo de la técnica "crisscroos", que implica el cruce intercambiado de progenitores, a fin de eliminar diferenciales a floración; ésto ya se ha confirmado con el híbrido H-137 (Tadeo, 1991).

Recientes investigaciones con el híbrido de maíz H137, han definido que al invertir el orden de cruza de sus progenitores al intercambiarIos, se facilita el proceso de producción de semilla. Una alternativa para solucionar el inconveniente de asincronía en este híbrido lo constituye la combinación (M37xM18) x (M36xM17), la cual presenta una diferencia a floración de únicamente un día, lo que mejora comparativamente la conformación original (M36xM37) x (MI7xMI8) de este híbrido (Tadeo, 1991). También se ha observado que el orden de la cruza inverso en la producción de semilla del híbrido H-137, es decir (MI7xMI8) x (M36xM37) además de elevar la productividad y calidad física de la semilla, presenta menor diferencial a floración entre progenitores que el orden de cruza directo (Espinosa y Tut, 1990; Tadeo y Espinosa, 1992; Espinosa y Tadeo, 1992).
Eckhart y Bryan (1940) citados por Jenkins (1978), señalan que puede haber diferencias significativas en rendimiento entre las tres cruzas dobles que pueden hacerse entre cuatro líneas endogámicas progenitoras; de manera similar se han registrado diferencias altamente significativas en el rendimiento de las cruzas dobles formadas de diferentes cruzas simples provenientes de cuatro líneas endogámicas (Coxtator y Johnson, citados por Sprague, 1955).

El orden in verso en el híbrido $\mathrm{H}$-137, adicionalmente a otras ventajas, favorece el manejo de fechas de siembra para aislar lotes de producción de semillas, algo difícil de conseguir en la Meseta Central, por la predominancia del monocultivo (Tadeo y Carballo, 1991).

En un estudio con las cruzas simples de híbridos de temporal (H-28, H-30, H-32), se definió que aún cuando no se presentaron efectos significativos, numéricamente hay tendencia favorable hacia un orden de cruza; para la cruza simple hembra del H-30, es decir H-34, híbrido simple comercial, se sugiere el orden recíproco (Hernández, 1989).

En este trabajo se evalúa la capacidad productiva del híbrido H-137 obtenido en cinco diferentes órdenes de combinación, además de dos versiones del híbrido en conformación trilineal.

El objetivo de este trabajo fue observar si hay diferencias del híbrido en su versión original, con respecto a versiones alternativas.

\section{MATERIALES Y MÉTODOS}

El trabajo se desarrolló durante el ciclo primaveravera-verano de 1991; se manejaron dos experimentos en Chapingo, México, y otro en Cuatitlán, México, en terrenos del Campo Experimental Valle de México (CEVAMEX) del INIFAP y Campo Experimental de la Facultad de Estudios Superiores Cuatitlán-UNAM, respectivamente. Ambas localidades se sitúan a una altura de 2240 msnm. 
El material gen ético empleado fue el híbrido H-137 obtenido en cinco órdenes diferentes de combinación de sus progenitores, además de dos cruzas de tres líneas:

1. (M17xM18) x (M36xM37);

2. (M37xM18) x (M36xM17);

3. (M36xM17) x (M37xM18);

4. (M36xM18) x (M37xM17);

5. (M36xM37) x (M17xM18);

6. (M17xM18) x M36;

7. (M17xM18) x M37.

En los tres ambientes se manejó un diseño experimental de bloques completos al azar; en dos con siete repeticiones y en el otro con tres.

El análisis estadístico se hizo de forma individual y posteriormente se estimaron las variables de estabilidad de acuerdo con Eberhart y Russel (1966).

En los tres experimentos la parcela total fue de cuatro surcos de $5 \mathrm{~m}$ de largo, con 0,80 m entre surcos y 0,50 m. entre matas; la parcela útil estuvo constituida por los dos surcos centrales. Se manejó una densidad aproximada a 60 mil plantas por hectárea en los tres experimentos.

Las variables evaluadas fueron: rendimiento, floración masculina y femenina, altura de planta y mazorca, pero hectolítrico del grano y peso de 200 semillas.

\section{RESULTADOS Y DISCUSIÓN}

En el Cuadro 1 se presentan los resultados de los análisis de varianza individuales para las variables evaluadas.

Para rendimiento solo hubo diferencia significativa en Chapingo, México (2), no así en los otros dos experimentos, lo cual concuerda en cierta medida con lo que señala Jenkins (1978).

En general se observaron pocas variables con significancia para el factor tratamientos.

Las comparaciones de medias para rendimiento, permiten detectar que los rendimientos más elevados, aún cuando sea numéricamente, de forma lógica correspondieron a las cruzas trilineales; el resto de los genotipos exhibieron valores similares entre ellos (Cuadro 2). Lo anterior se ratifica con los resultados del análisis de varianza (Cuadro 3), para la estimación de los parámetros de estabilidad, que definieron desigualdad en rendimientos entre variedades, pero en todos los casos, los genotipos se clasificaron como estables, de acuerdo con Carballo (1970).

En promedio destacaron por su productividad, aunque como ya se anotó, numéricamente, las dos combinaciones trilineales (M17xM18) x M37 y (M17xM18) x M36; la cruza original (M36xM37) x (M17xM18) fue superada numéricamente por la cruza recíproca y

Cuadro 1. Cuadrados medios de análisis de varianza, individuales para variables evaluadas en el híbrido de maíz H-137, bajo diferente orden de cruza.

\begin{tabular}{|c|c|c|c|c|c|c|c|c|c|c|c|c|}
\hline \multirow[b]{2}{*}{ Variable } & \multicolumn{3}{|c|}{ Chapingo, Méx. (2) } & \multirow[b]{2}{*}{ Media } & \multicolumn{3}{|c|}{ Chapingo, Méx. (1) } & \multirow[b]{2}{*}{ Media } & \multicolumn{3}{|c|}{ Cuatitlán, Mex. } & \multirow[b]{2}{*}{ Media } \\
\hline & Trat. & Rep. & C.V. & & Trat. & Rep. & c.v. & & Trat. & Rep. & C.v. & \\
\hline Rendimiento & $4,52 *$ & 2,82 & 17,6 & 7,078 & 3,45 & $20,88^{* *}$ & $26-1$ & 6,7 & 1,92 & $6,3^{* *}$ & 23,5 & 5,279 \\
\hline Floración masculina & 28,23 & 31,57 & 7,0 & 85 & $22,43^{* *}$ & $22,43 * *$ & $1-7$ & 86 & $8,99 *$ & $23,85^{* *}$ & 2,0 & 93 \\
\hline Floración femenina & 3,41 & 1,08 & 1,5 & 87 & $25,01^{* *}$ & 2,58 & $1-7$ & 88 & $5,85^{* *}$ & $11,9^{* *}$ & 1,3 & 97 \\
\hline Altura de planta & $434,89 * *$ & 63,31 & 3,4 & 283 & 290,17 & 94,02 & $4-7$ & 285 & 952,08 & 2043,36 & 17,0 & 253 \\
\hline Altura de mazorca & 198,27 & $261,94 *$ & 5,3 & 189 & 142,75 & 49,56 & $5-3$ & 188 & 113,11 & $324,40 * *$ & 4,8 & 146 \\
\hline Peso hectolítrico & 131,33 & 80,57 & 1,0 & 792 & 412,70 & 1,56 & $1-8$ & 780 & 626,2 & 904,0 & 3,0 & 672 \\
\hline Peso de 200 sem. & $171,89^{* *}$ & $106,33^{*}$ & 9,4 & 68,8 & 135,27 & 129,64 & $11-9$ & 68,9 & $121,5^{*}$ & 66.69 & 11.9 & 55,9 \\
\hline
\end{tabular}


Cuadro 2. Rendimieto de grano en tres ambientes de evaluación del híbrido de maíz H-137 bajo diferente orden de cruza.

\begin{tabular}{llccc}
\hline $\begin{array}{l}\text { No. de } \\
\text { Trat. }\end{array}$ & Orden de combinación & $\begin{array}{c}\text { Chapingo, Méx. (2) } \\
\text { Rend (kg/ha) }\end{array}$ & $\begin{array}{c}\text { Chapingo, Méx. (1) } \\
\text { Rend (kg/) }\end{array}$ & $\begin{array}{c}\text { Cuatitlán, Méx. } \\
\text { Rend (kg/ha) }\end{array}$ \\
\hline 1 & (M17xM18) x (M36xM37) & $7,381 \mathrm{ab}$ & 5,444 & 5,228 \\
2 & (M37xM18) x (M36xM17) & $6,160 \mathrm{ab}$ & 6,340 & 5,169 \\
3 & (M36xM17) x (M37xM18) & $7,138 \mathrm{ab}$ & 6,514 & 4,930 \\
4 & (M36xM18) x (M37xM17) & $7,169 \mathrm{ab}$ & 6,529 & 4,777 \\
5 & (M36xM37) x (M17xM18) & $5,876 \mathrm{~b}$ & 5,197 & 4,888 \\
6 & (M17xM18) x M36 & $7,691 \mathrm{ab}$ & 7,048 & 6,217 \\
7 & $($ M17xM18) x M37 & $8,132 \mathrm{a}$ & 8,548 & 5,749 \\
\hline D.S.H. $(0,05)$ & 2,085 & 5,357 & 2,072
\end{tabular}

Cuadro 3. Cuadrados medios de los análisis de varianza en la estimación de parámetros de estabilidad para variables evaluadas en el híbrido de maíz H-137 bajo diferente orden de combinación de progenitores.

\begin{tabular}{|c|c|c|c|c|c|c|c|c|}
\hline \multirow{3}{*}{$\begin{array}{l}\text { Fuente de } \\
\text { variación }\end{array}$} & \multirow{2}{*}{\multicolumn{2}{|c|}{ Rendimiento }} & \multicolumn{2}{|c|}{ Floración } & \multicolumn{2}{|c|}{ Altura } & \multirow{3}{*}{$\begin{array}{c}\text { Peso hectolítrico } \\
\text { C. } \text { M. }\end{array}$} & \multirow{3}{*}{$\begin{array}{l}\text { Peso } 200 \\
\text { C. } M .\end{array}$} \\
\hline & & & \multirow{2}{*}{$\begin{array}{l}\text { Masc. } \\
\text { C.M. }\end{array}$} & \multirow{2}{*}{$\begin{array}{l}\text { Fem. } \\
\text { C.M. }\end{array}$} & \multirow{2}{*}{$\begin{array}{l}\text { Planta } \\
\text { C.M. }\end{array}$} & \multirow{2}{*}{$\begin{array}{l}\text { Mazorca } \\
\text { C.M. }\end{array}$} & & \\
\hline & G.L. & C.M. & & & & & & \\
\hline Variedades (V) & 20 & $1,55^{*}$ & 2,08 & $1,86^{*}$ & $145,52 *$ & $55,43 *$ & 67,87 & $1720632,06 *$ \\
\hline Ambientes (A) & 6 & & & & & & & \\
\hline \multicolumn{9}{|l|}{$\mathrm{V} \times \mathrm{A}$} \\
\hline A (lineal) & 1 & & & & & & & \\
\hline V x A (lineal) & 6 & 0,24 & 0,75 & 0,06 & $106,68^{*}$ & 22,61 & 94,11 & $3449409,47 *$ \\
\hline Desviación conjun & inta 7 & 0,31 & 4,19 & 0,68 & 28,56 & 22,50 & 72,14 & 1592,31 \\
\hline Variedad 1 & 1 & 1,02 & 0,89 & 1,21 & 34,01 & 0,11 & 25,31 & $213,64 *$ \\
\hline Variedad 2 & 1 & 0,15 & 1,25 & 0,17 & 64,78 & 6,24 & 181,97 & $9030,47 *$ \\
\hline Variedad 3 & 1 & 0,00 & 0,15 & 1,21 & 8,17 & 21,47 & 247,57 & $269,26 *$ \\
\hline Variedad 4 & 1 & 0,01 & $21,22 *$ & 0,10 & 0,00 & 53,36 & 21,33 & 143,62 \\
\hline Variedad 5 & 1 & 0,09 & 1,25 & 0,10 & 25,16 & 1,08 & 15,36 & $96,06 *$ \\
\hline Variedad 6 & 1 & 0,02 & 1,17 & 0,99 & 4,91 & 28,22 & 1,76 & 27,75 \\
\hline Variedad 7 & 1 & 0,86 & 3,40 & 0,99 & 62,91 & 47,00 & 11,66 & $1365,36^{*}$ \\
\hline Error conjunto & & 0,46 & 2,20 & 0,39 & 117,04 & 17,53 & 45,43 & 11,06 \\
\hline Total & 20 & & & & & & & \\
\hline
\end{tabular}

*, ** Significativo al nivel de 5 y $1 \%$ de probabilidad de error. 
combinaciones alternativas, lo cual concuerda con la propuesta de Tadeo (1991), así los señalamientos de Espinosa y Tut (1990).

Los resultados encontrados al aplicar los parámetros de estabilidad indican similitud en la clasificación de estabilidad de la combinación original con el orden inverso, así como por lo menos una combinación alternativa y ambas cruzas trilineales; lo anterior es importante si se toma en cuenta que además de asegurarse buena productividad de grano del híbrido, se facilita la sincronía a floración.

La combinación original del híbrido H-137 o sea (M36xM37) x (M 17xM18) quedaría descartada como opción viable de producción de semillas, pués además de que fue la menos rendidora, de manera numérica, mostraron alto diferencial a floración entre sus progenitores (Cuadro 4).

Las combinaciones trilineales podrían ser una buena opción, ya que además de su productividad de grano, presenta ventajas en la producción de semilla, al ser menos complicada su conformación y más sencillo mantener su calidad genética.

De acuerdo con los objetivos trazados en esta investigación, se confirma que la combinación alternativa (M37xM18) x (M36xM17) es una excelente posibilidad para hacer factible y redituable el establecimiento de programas de producción de semilla del híbrido H-137, ya

Cuadro 4. Medias y parámetros de estabilidad evaluadas en el híbrido de maíz H-137 bajo diferente orden de combinación de progenitores.

\begin{tabular}{|c|c|c|c|c|c|c|c|c|c|c|c|c|}
\hline \multirow{2}{*}{$\begin{array}{l}\mathrm{N}^{\circ} \text { de Orden de combinación } \\
\text { Trat. }\end{array}$} & \multicolumn{4}{|c|}{ Rendimiento } & \multicolumn{4}{|c|}{ Floración maxc. } & \multicolumn{4}{|c|}{ Floración Fem. } \\
\hline & Media & bi & $\mathrm{S}^{2} \mathrm{di}$ & $\mathrm{C}^{1 /}$ & Media & bi & $\mathrm{S}^{2} \mathrm{di}$ & $\mathrm{C}^{1 /}$ & Media & bi & $S^{2} d i$ & $\mathrm{C}^{1 /}$ \\
\hline$(\mathrm{M} 17 \times \mathrm{M} 18) \times(\mathrm{M} 36 \times \mathrm{M} 37)$ & 6,02 & 1,03 & 0,56 & 1 & 86 & 0,78 & $-1,31$ & 1 & 89 & 0,97 & 0,82 & 1 \\
\hline$(\mathrm{M} 37 \times \mathrm{M} 18) \times(\mathrm{M} 36 \mathrm{x} 17$ & 5,89 & 0,62 & $-0,31$ & 1 & 88 & 0,98 & $-0,95$ & 1 & 90 & 1,03 & 0,22 & 1 \\
\hline (M36; & & 1,24 & $-0,46$ & 1 & 88 & 1,04 & $-2,05$ & 1 & 91 & 0,97 & 0,82 & 1 \\
\hline (M36 & & 1,34 & $-0,45$ & 1 & 87 & 1,19 & $19,02 *$ & 2 & 91 & 0,98 & $-0,29$ & 1 \\
\hline (M36 & & 0,50 & 0,37 & 1 & &, 98 & 0,95 & 1 & 91 & 0,98 & $-0,29$ & 1 \\
\hline (M17xM18) x M36 & 99 & 0,80 & $-0,44$ & 1 & 87 & 1,10 & $-1,03$ & 1 & 89 & 1,04 & 0,60 & 1 \\
\hline (M17x) & 7,48 & 1,48 & 0,40 & 1 & 87 & 0,93 & 1,20 & 1 & 90 & 1,04 & 0,60 & 1 \\
\hline D.S.H. $(0$ & & & & & 6 & & & & 3 & & & \\
\hline \multirow{2}{*}{$\begin{array}{l}N^{o} \text { de Orden de combinación } \\
\text { Trat. }\end{array}$} & \multicolumn{4}{|c|}{ Altura de planta } & \multicolumn{4}{|c|}{ Altura de mazorca } & \multicolumn{4}{|c|}{ Peso hectolítrico } \\
\hline & Media & bi & $S^{2} \mathrm{di}$ & $\mathbf{C}^{1 /}$ & Media & bi & $S^{2} \mathrm{di}$ & $\mathrm{C}^{1 /}$ & Media & bi & $S^{2} d_{i} C^{1}$ & $\mathrm{C}^{1 /}$ \\
\hline (M17xM & & & $-83,03$ & 31 & 175 & 0,93 & $-17,42$ & 1 & 742 & 1,09 & $-20,12$ & 1 \\
\hline$(\mathrm{M} 37 \mathrm{xM} 18) \times(\mathrm{M} 36 \mathrm{xM} 17)$ & 267 & 1,24 & $-52,26$ & 61 & 168 & 0,93 & $-11,29$ & 1 & 743 & 0,94 & 136,54 & 2 \\
\hline$(\mathrm{M} 36 \times \mathrm{M} 17) \times(\mathrm{M} 37 \mathrm{xM} 18)$ & & 0,92 & $-108,87$ & 71 & 172 & 0,97 & 3,94 & 1 & 748 & 1,11 & 202,14 & 2 \\
\hline$(\mathrm{M} 36 \times \mathrm{M} 18) \times(\mathrm{M} 37 \times \mathrm{M} 17)$ & 279 & $0,20 *$ & $-117,04$ & 43 & 172 & 1,17 & 35,83 & 1 & 757 & 0,98 & $-24,10$ & 1 \\
\hline (M36xM37 & 27 & 1,29 & $-91,88$ & 81 & 175 & 0,92 & $-16,45$ & 1 & 747 & 0,82 & $-30,07$ & 1 \\
\hline$(\mathrm{M} 17 \mathrm{xM} 18) \times \mathrm{M} 36$ & 278 & 0,94 & $-112,13$ & 31 & 174 & 0,85 & 10,69 & 1 & 750 & 0,95 & $-43,67$ & 1 \\
\hline (M17xM18) x M37 & 283 & 1,27 & $-54,13$ & 31 & 182 & 1,22 & 29,47 & 1 & 749 & 1,09 & $-33,77$ & 1 \\
\hline
\end{tabular}

" De acuerdo con Carballo (1970): 1= Estable, 2= Buena respuesta en todos los ambientes pero inconsistente; $3=$ Respuesta mejor en ambientes desfavorables y consistentes; $4=$ Respuesta mejor en ambientes desfavorables pero inconsistente; $5=$ Buena respuesta en buenos ambientes. Y consistente; $6=$ Mejor respuesta en ambientes buenos pero inconsiente. 
que coincide totalmente en la floración de sus progenitores, exhibe buena capacidad productiva de grano y además demostró estabilidad.

\section{CONCLUSIONES}

El cambio de orden de progenitores en el proceso de producción de semilla del híbrido H-137, no propició efectos significativos sobre la expresión de rendimiento y su estabilidad, se observó similar capacidad productiva y estabilidad, independientemente del orden de cruza utilizado.

La producción de semilla bajo el orden (M37xM18) x (M36xM17), además de facilitar la siembra simultánea de progenitores, asegura buena productividad del híbrido H-137, así como calidad de semilla, lo cual la define como el mejor orden de cruza; bajo este esquema de combinación, es factible la siembra simultánea de progenitores y además se mantienen las características favorables del H-137.

Las combinaciones trilineales (M17xM18)x M36 y (M17xM18)xM37, supera numéricamente al resto de combinaciones del H-137. La conformación trilineal representa genotipos diferentes al H-137 y podrían ser una opción para mayor productividad de grano. Convendrá evaluar estos materiales en otros ambientes, a fin de determinar mejor su potencial productivo.

\section{LITERATURA CITADA}

ASTEINZA, B. G.; F. SOLIS M.; A. ESPINOSA C. 1990. Efec-

to de la aplicación de gapol y ethrel en la floración masculina para producción de semilla del híbrido de maíz H137. In: Resúmenes del XIII Congreso Nacional de Fitogenética. SOMEFI, Escuela Superior de Agricultura "Hermanos Escobar" Cd. Juárez, Chih.

CARBALlO, C., A. 1970. Comparación de variedades de maíz de El Bajío y de la Mesa Central por su rendimiento y estabilidad tesis M.C. ENA, Colegio de Postgraduados, Chapingo, México.
EBERHART, S. A.; W. A. RUSSELL. 1966. Stability parameters for camparing varieties, Crop. Sci. 6:36-40.

ESPINOSA C., A.; C. TUT Y C. 1990. Tecnología de producción de semillas del híbrido de cruza doble de maíz H-137 de Valles Altos. In: Resúmenes del XIII Congreso Nacional de Fitogénetica, SOMEFI, Escuela Superior de Agricultura Hermanos Escobar, Cd. Juárez, Chih.

ESPINOSA c., A.; M. TADEO R 1992. Producción de semilla del híbrido doble de maíz H-137 en respuesta a la fertilización y densidad de población. In: Revista Fitotecnia Mexicana. Vol. 15(1): 1-10.

HERNÁNDEZ C., A. 1989. Influencia del orden de cruzamiento de los progenitores en el rendimiento y calidad de semilla del híbrido de maíz de temporal. Tesis de licenciatura, FESC, UNAM. Cuautitlán, Izcalli, Méx.

JENKINS, M. T. 1978. Predicting hibrid performance. In: Maize breeding during the development of hibrid maize. Ed. Wiley Intenscience. cap. 2.

PIÑA, D. V.; M. TADEO, R.; A. ESPINOSA C. 1992. Capacidad productiva de híbridos de maíz por diferente orden de combinación de progenitores. In: Resúmenes del XIV Congreso Nacional de Fitogenética SOMEFI, Escuela de Ciencias Agronómicas, Campus V, Universidad Autónoma de Chiapas, Tuxtla Gutierrez, Chis.

SPRAGUE, G. F. 1955. Corn and improvement. Ed. Academic Press. American Society Agronomy. Vol 5.

TADEO R, M. 1991. Producción de semillas en híbridos de maíz con problemas de sincronía en la floración de sus progenitores. Tesis de M. C. Colegio de Postgraduados.

TADEO R, M.; A. CARBALLO C. 1993. Producción de semillas en híbridos de maíz con problemas de sincronía en la floración de sus progenitores. In: Agrociencia, Colegio de Postgraduados, Montecillo México. 